\title{
GROWTH AND YIELD OF ZUCCHINI TYPE SUMMER SQUASH \\ (CUCURBITA PEPO L.) FERTILIZED BY COMBINED AZOTOBACTER CHROOCOCUM MUTANTS AND MINERAL N-FERTILIZATION
}

\author{
REFAI, E. F. ${ }^{1}$, H. FOLY ${ }^{1}$ AND O.F. DAKHLY ${ }^{2}$ \\ 1. Agriculture Research Center, Horticulture Institute, Giza \\ 2. Faculty of Agriculture, Minia University, Giza
}

(Manuscript received 23 November 2009)

\begin{abstract}
Summer squash (Cucurbita pepo L.) zucchini type cv "Eskandarany" grown in reclaimed sandy soil at the Horticulture Research Station, Arab El-Awammer, Assiut Governorate, Egypt was fertilized with nitrogen $(0,30,60,90$ and 120 units / feddan) from ammonium nitrate $\left(\mathrm{NH}_{4} \mathrm{NO}_{3}\right)$ after being either inoculated with one of Azotobacter chroococum strains (5 mutants and wild type strain) or without inoculation. Azotobacter chroococum mutant strains were induced by $\mathrm{N}$ - methyl $\mathrm{N}$ - nitro $-\mathrm{N}$-nitrosogaunidine (NTG) treatment for suspension of the wild type cells in Microbial Genetics Lab. at the Department of Genetics, Minia University. Assessment of plant growth, development and yield indicated that application of $120 \mathrm{~N}$ units/feddan gave the longest stem, greatest number of leaves, female flowers and immature fruits and early and total yield as compared to other sole $\mathrm{N}$ applications. Biofertilization using Azotobacter wild type strain resulted in a significant increment in stem length, number of female and male flowers, number and size of immature fruits and early and total yield under the conditions of $\mathrm{N}$ fertilizer application up to 120 units per feddan. Number of leaves and female flowers showed similar result all $\mathrm{N}$ fertilization levels but not $120 \mathrm{~N}$ units. In general, use of induced Azotobacter mutants resulted in an increase in all studied parameters comparing with the use of wild type strain. One of the Azotobacter mutant strains (denoted \#7) seemed to be the best among the others for enhancing plant growth, development and yielding. This Azotobacter mutant strain combined with 90 mineral $\mathrm{N}$ units had $5.5 \%$ increase over the yield produced with sole $120 \mathrm{~N}$ units, thus saving one quarter of the mineral $\mathrm{N}$ amount added. It is concluded that combined utilization of Azotobacter chroococum and mineral $\mathrm{N}$-fertilizer could enhance productivity of summer squash in new reclaimed sandy soil and mutation could be employed as potential approach to elevate the efficiency of this bacterium species as a bio-fertilizer.
\end{abstract}

\section{INTRODUCTION}

Squash (Cucurbita pepo L.) is one of the most popular vegetable crops grown in Egypt. In most monoecious cucurbit plants, the ratio of staminate to pistillate flowers greatly varies when the plants are grown under different environmental conditions, including photoperiod, temperature, nutrient availability, or exogenous treatment with 
plant hormones (Lau and Stephenson, 1993, Swiader et al., 1994, Yin and Quinn, 1995).

Biological fertilization by $\mathrm{N}_{2}$ fixing bacteria has recently received a significant attention in production of crop plants. The efficient use of bio-fertilizers may be affected by different strain groups such as nitrogen fixer and nutrient mobilization microorganisms which help in increasing the availability of minerals and their forms in the composted materials and increase levels of extractable macro- or micronutrients (El-Karamany et al., 2000).

Enhanced productivity of different crop plant in wheat , El-Metwaly, 1998, in pepper (Capiscum annuum L.) Abdalla et al., 2001, in Cantaloupe(Cucumis melo) Adam et al. 2002) as result of bio-fertilizer application has been reported. Abd ElFattah and Sorial (2000), on summer squash, indicated that bio-fertilizer treatment (Halex2) significantly enhanced the induction of female flowers, which was reflected afterward on the increase of fruit yield.

It is widely documented that a considerable number of bacterial species, mostly those associated with the rhizosphere, are able to exert a beneficial effect on plant growth. These bacteria include strains in the genera Bacillus, Pseudomonas, Rhizobium and others have been called 'plant growth regulator promoting rhizobacteria' (PGPR) (Bloemberg and Lugtenberg, 2001). PGPR bacteria can stimulate growth and yield of crop species including potato, radish, tomato, lettuce, beans, cucumber (De Silva et al., 2000). All the monitored activities and formulation properties suggest an effective use of Bacillus subtilis as a plant -strengthening agent and/or bio-control of diseases.

Nowadays, bio-fertilizers are considered one of the top biotechnology applications for establishing productive non-polluting organic agriculture (Shehata, and El- Khawas, 2003). It can help to overcome the ecological problems resulting from the loss of plant nutrients and provide sustainable solutions for present and future agricultural practices (Rai, 2006). Completely fermented organic matters resulted in bio-fertilizers which improve the physical properties of soils leading to better aeration and water and nutrient retention capacity.

Free living $\mathrm{N}$-fixing bacteria are associated with roots of many cereal crops and have beneficial effects on crops yield (Neyra and Dobereiner ,1977). A remarkable increase in the plant fresh and dry weight and nitrogen content in plant and soil has been achieved when used either Azotobacter vinelndii wild type strain or its mutants 
(AbdelRaheem et al., 1995). The present research study investigates nitrogen fixation utilizing Azotobacter chroococum induced biochemical mutants aiming to obtain more efficient strains for production of summer squash towards reducing mineral $\mathrm{N}$ application in production of summer squash.

\section{MATERIALS AND METHODS}

\section{Laboratory study}

Azotobacter chroococum wild type strain was isolated from rhizosphere around roots of the squash plants cultivated in the farm of Agricultural Faculty, Minia University, Mania, Egypt (Dakhly and Abedel-Mageed, 1997). A complete media (CM) was used for Azotobacter chroococum culturing (Strandberg and Wilson 1968). Minimal medium (MM) was used to isolate the auxotrophic of Azotobacter chroococum (Mchenney and Melton, 1986). All media were autoclaved at $121^{\circ} \mathrm{C}$ for $20 \mathrm{~min}$.

Cells of Azotobacter chroococum were treated with 0.0, 0.2, 0.4, 0.6, 0.8 and $1.0 \mathrm{mg} \mathrm{N}$ - methyl $\mathrm{N}$ - nitro $-\mathrm{N}$-nitrosogaunidine (NTG) $\mathrm{mg} / \mathrm{ml}$ of cell suspension for one and two $\mathrm{h}$ at $30 \mathrm{C}^{\circ}$. A. chroococum wild type single colonies were subculture on slants of complete medium and incubated at $30^{\circ} \mathrm{C}$ for 3 days. One loop from subculture was added to $5 \mathrm{ml}$ sterilized distilled water in a test tube. One $\mathrm{ml}$ sample of cell suspension were distributed on sterilized test tubes. The final concentrations of $\mathrm{N}$ methyl $\mathrm{N}$ - nitro $-\mathrm{N}$-nitrosogaunidine (NTG) were $0.0,0.20,0.40,0.60,0.80$ and 1.0 $\mathrm{mg} / \mathrm{ml}$. The suitable dilutions from each concentration were plated on complete media using six plants for each concentration. The plates were incubated at $30^{\circ} \mathrm{C}$ for three days and the surviving colonies were counted. Single colonies were tested on $\mathrm{MM}$ and $\mathrm{CM}$ and incubated at $30^{\circ} \mathrm{C}$ for 5 days. Bacterial growth was compared on MM and $\mathrm{CM}$ and the mutants were selected. The selected mutants were test for their specific single or double requirements by applying Holliday (1960) system.

\section{Field study}

Field experiment was carried out during two successive growing seasons at the Farm of Agriculture experiments, Horticulture Research Station, Arab El-awammer, Assiut Governorate, Egypt. Summer squash (Cucurbita pepo L.) zucchini type cv "Eskandarany" seeds were sown on Sept.10 and 20 in 2008 and 2009, respectively. Five application rates of nitrogen fertilizer from ammonium nitrate $\left(\mathrm{NH}_{4} \mathrm{NO}_{3}\right)(0,30$, 60,90 and 120 units of nitrogen) were studied under conditions of plant inoculation 
with either one of five Azotobacter mutants, wild type strain and without inoculation. Each N-level was divided into three equal doses, and applied during field preparation then after 20 and 40 days from seed sowing. The treatments were arranged in a splitplot design with three replicates. The $\mathrm{N}$-fertilizer rate was in the main plot and the Azotobacter mutants in sub-plot. The plot area was $12.8 \mathrm{~m}^{22}$ and consisted of four rows each $4 \mathrm{~m}$ long and $0.8 \mathrm{~m}$ wide. Plants were spaced $40 \mathrm{~cm}$ apart on one side of the ridge. The cultural practices were done in accordance with those advised for summer squash production.

A random sample of three plants from each treatment was used for evaluating main stem length (cm, 45 days after sowing), number of leaves/plant, number of produced female and male flowers, average fruit length and diameter $(\mathrm{cm})$ and average fruit weight $(\mathrm{g})$. During the production season, fruits were harvested at two days intervals, counted and then weighed and number of fruits/plant was recorded. Early yield was determined from the early 4 harvests, whereas the average total yield was recorded during the whole harvesting period (Ton/Feddan). Data were statistically processed following the procedure of analysis of variance (Gomez and Gomez, 1984) and means were compared using "The Least Significant Difference Test" (LSD) at 0.05 probability level.

\section{RESULTS AND DISCUSSION}

\section{Laboratory study}

Data in Table (1) show the effect of different $\mathrm{N}$ - methyl $\mathrm{N}$ - nitro $-\mathrm{N}$ nitrosogaunidine concentrations $(0.0,0.2,0.4,0.6,0.8$ and $1.0 \mathrm{mg} / \mathrm{ml}$ of cell suspension) for 1 and $2 \mathrm{~h}$ at $30^{\circ} \mathrm{C}$ on Azotobacter chroococum. The percentage of wild type survival tended to decline (100, 70, 55, 42, 30 and 19) with increasing concentration of mutagenic agent $(0.0,0.2,0.4,0.6,0.8$ and $1.0 \mathrm{mg} / \mathrm{ml})$ for $1 \mathrm{~h}$ exposure time. A sharper decline $(100,60,40,18.7$.and $0.2 \%)$ was observed in percent survival when subjected to mutagenic agent for two $h$. For $1 \mathrm{~h}$ subjection time, $25(1.56 \%)$ out of 1643 tested colonies proved to be mutants. They were identified as four alanineless, two valeneless, two adenineless argenineless, three histidineless, one threonineless, two methionineless, two prolineless, two treptophanelss and six reucrtant. For 2 h subjection time, 34 (2.34 \%) out of 1489 were identified as mutants, three alanineless, three valeneless, two adenineless, two 
argenineless, three histidineless, three threonineless, two methionineless, three prolineless, four treptophanelss, three phenylalanineless, three tyrosineless and three revertant mutants. It can be observed that 0.8 and $1.0 \mathrm{mg}$ (NTG)/ $\mathrm{ml}$ induced an elevated frequency of mutation whether Azotobacter chroococum cells were subjected for 1 or 2 hours. Similar results were found in Azotobacter by Bishop et al (1980), Abdel-Raheem et al. (1995), Dakhly et al., (1998), Kumar and Norula (1999), Mahmoud, (2000) and Hassan et al. (2000).

\section{Field study}

The effect of mineral nitrogen fertilizer and bio-fertilization with Azotobacter chroococum wild and mutant strains on stem length, number of leaves, male and female flower and immature fruits per plant, early and total yield per plant, and fruit length, diameter and weight of zucchini type summer squash cv. "Eskandarany" are presented in Tables (2, 3 and 4). Plants received 30, 60, 90 and 120 units/fed. mineral nitrogen fertilizer had longer stem, increased number of leaves, and produced greater number of female flowers (Table 2) and immature fruits (Table 3) compared with untreated plants (plants grown without mineral or bio-fertilizers, negative control). While we practiced harvest at regular intervals, there were an increase in fruit length, diameter and weight (Table 4). It is noticeable, that squash plants in this study showed a significant increase in number of male flowers at 30 and $60 \mathrm{~N}$ units (Table 2) reflecting the overall growth vigor. However, it decreased then stabilized afterwards at 90 and $120 \mathrm{~N}$ units as the plant requirements of $\mathrm{N}$ were balanced. Availability of male flowers is regarded useful as to provide pollination to set fruit from female ones. Indeed those plants produced higher early and total yield (Table 3).

Besides day length and temperature, it is well documented that summer squash plants cv. "Eskandrany" positively respond to availability of $\mathrm{N}$ in terms of enhanced growth, female flowers development and fruit yield (Lau and Stephenson, 1993, Swiader et al., 1994, Yin and Quinn, 1995, Abd El-Fattah and Sorial, 2000, Refai and Mohamed, 2009). This is especially true in low fertility medium such as reclaimed soil used in this study (Abd El-Fattah and Sorial (2000). Obviously, the longest stem, greatest number of leaves, female flowers and immature fruits and early and total yield were obtained here when plants were fertilized with $120 \mathrm{~N}$ units/feddan. In agreement with other studies conducted using zucchini type summer squash cv. "Eskandrany", femininity leading to increased harvested immature fruits is a major 
component of fruit early and total yield (Goicoechea et al., 1995, Mohamed, 1996, Noel et al., 1996, Refai and Mohamed, 2009).

Bio-fertilization using Azotobacter wild type strain for squash plants that received no mineral $\mathrm{N}$ fertilizer resulted in a significant increment in stem length, number of leaves, female flowers and immature fruits, early and total yield of larger fruits (length, diameter and weight) but reduced number of male flowers. Such result existed also for stem length, number of female and male flowers, number and size of immature fruits and early and total yield under the conditions of $\mathrm{N}$ fertilizer application up to 120 units per feddan. Number of leaves and female flowers showed similar response under conditions of $\mathrm{N}$ fertilization in the range from 30 to $90 \mathrm{~N}$ units but not $120 \mathrm{~N}$ units per feddan. There were significant differences among the Azotobacter biofertilizer treatments. With few exceptions, use of induced Azotobacter mutants in this study resulted in longer stem, increased number of leaves, female flowers and immature fruits and reduced number of male flowers and elevated early and total yield comparing with the use of wild type strain. Obviously, Azotobacter mutant strain 7 seemed to be the best among the other mutants for enhancing summer squash plant growth, development and yielding. Utilization of Azotobacter wild strain combined with $60 \mathrm{~N}$ and 90 units produced $40.7 \%$ and $15.8 \%$ reduction, respectively, in fruit yield compared to using sole $120 \mathrm{~N}$ units. Worthwhile to mention, that this reduction was narrowed to $15.8 \%$ when used Azotobacter mutant strain 7 combined with $60 \mathrm{~N}$ units. An increase of $5.5 \%$ over the yield produced with sole $120 \mathrm{~N}$ units was obtained when used Azotobacter mutant strain 7 combined with $90 \mathrm{~N}$ units. This shows that Azotobacter mutant strain 7 can save one quarter of the mineral $\mathrm{N}$ amount while realizing higher yielding. Such reduction in mineral $\mathrm{N}$ application means saving a quarter of the energy need in $\mathrm{N}$ fertilizer industry besides conserving our living environment and reducing negative effects leading to undesirable climatic changes.

In the present study, we partitioned mineral $\mathrm{N}$ application to several sequential doses to maximize uptake via reducing its possible leach. Interestingly, however, Azotobacter tend to show an enhancing effects on growth, development and fruit yielding of summer squash at relatively high level of mineral $\mathrm{N}$ applications. A similar simulative effect for this bacterium on growth and development were previously reported by others (Bochow and Dolej, 1999 and Adam et al. (2002)). Such simulative effect may be due to its added $\mathrm{N}$ fixation for the growing plants (AbdelRaheem et al. (1995). In such regard, Azotobacter would be beneficial in terms of reducing further 
mineral $\mathrm{N}$ application that may not be desired as far as the human health and environment conservation is concerned. However, it is suggested (De Silva et al., 2000, Sudhakar et al., 2000) that the mechanism seem also to be based on hormons due to releasing exogenous bacterial metabolites having precursors of auxin (indole-3pyruvic acid), or inducers (GA3 fraction) for auxin synthesis. Many other investigators showed that such bacterial inoculation of seeds or roots leads to changes in plant growth which is caused by growth regulating substances especially those of gibberelin, cytokinin and IAA (Goicoechea et al., 1995, Noel et al., 1996). The bacterial biofertilizer application might then promote the crop growth by increasing root number and root length. Subsequently, root system can absorb more water and nutrients from soil including the applied $\mathrm{N}$. Thus, $\mathrm{N}$ lose hazards to the environment is reduce, especially, in reclaimed/sandy soil.

Overall results of this study suggest that combined utilization of Azotobacter chroococum and mineral $\mathrm{N}$-fertilizer could enhance productivity of summer squash in new reclaimed sandy soil. Mutation could be a potential approach to elevate the efficiency of this bacterium species as a bio-fertilizer.

\section{ACKNOWLEDGMENT}

The authors would like to thank Prof. Dr. Mohamed Fouad Mohamed for his scientific advice and critical reading of this manuscript. 
Table 1. Mutagenic effect of $\mathrm{N}$ - methyl $\mathrm{N}$ - nitro $-\mathrm{N}$-nitrosogaunidine on Azotobacter chroococum wild type.

\begin{tabular}{|c|c|c|c|c|c|c|c|c|c|c|c|c|c|c|c|c|c|c|}
\hline \multirow{2}{*}{$\begin{array}{c}\text { Exposure } \\
\text { time }\end{array}$} & \multirow{2}{*}{$\begin{array}{c}\text { Mutagen } \\
\text { Conc. } \\
\text { mg/ml }\end{array}$} & \multicolumn{2}{|c|}{ Survivals } & \multirow{2}{*}{$\begin{array}{l}\text { No .of } \\
\text { colonies } \\
\text { testing }\end{array}$} & \multirow{2}{*}{$\begin{array}{l}\text { No. of } \\
\text { mutants }\end{array}$} & \multirow{2}{*}{$\begin{array}{c}\text { Mutation } \\
\text { frequency \% }\end{array}$} & \multicolumn{12}{|c|}{ Requirements of single mutants } \\
\hline & & No. & $\%$ & & & & Ala- & $\mathrm{Val}^{-}$ & $\mathrm{Ad}^{-}$ & Arg & His & Thr- & Meth+- & Pro $^{-}$ & Trep- & Phoala- & Tyr $^{-}$ & Rever \\
\hline \multirow{7}{*}{1 hour } & 0.00 & 100000 & 100 & 250 & 0.00 & 0.00 & 0.0 & 0.0 & 0.0 & 0.0 & 0.0 & 0.0 & 0.0 & 0.0 & 0.0 & 0.0 & 0.0 & 0.0 \\
\hline & 0.20 & 70000 & 70.0 & 300 & 4.00 & 1.33 & 1 & 0.0 & 0.0 & 0.0 & 1 & 0.0 & 0.0 & 0.0 & 0.0 & 0.0 & 0.0 & 2 \\
\hline & 0.40 & 55000 & 55.0 & 280 & 3.00 & 1.07 & 0.0 & 1 & 0.0 & 0.0 & 0.0 & 1 & 0.0 & 0.0 & 1 & 0.0 & 0.0 & 0.0 \\
\hline & 0.60 & 42000 & 42.0 & 320 & 5.00 & 1.56 & 0.0 & 0.0 & 2 & 0.0 & 1 & 0.0 & 0.0 & 0.0 & 1 & 0.0 & 0.0 & 1 \\
\hline & 0.80 & 30000 & 30.0 & 220 & 7.00 & 3.18 & 2 & 0.0 & 0.0 & 1 & 0.0 & 0.0 & 2 & 1 & 0.0 & 0.0 & 0.0 & 1 \\
\hline & 1.00 & 19000 & 19.0 & 273 & 6.00 & 2.20 & 1 & 1 & 0.0 & 0.0 & 1 & 0.0 & 0.0 & 1 & 0.0 & 0.0 & 0.0 & 2 \\
\hline & Total & & & 1643 & 25 & 1.56 & 4 & 2 & 2 & 1 & 3 & 0.0 & 2 & 2 & 2 & 0.0 & 0.0 & 6 \\
\hline \multirow{7}{*}{2 hour } & 0.00 & 100000 & 100 & 270 & 1.00 & 0.37 & 1 & 0.0 & 0.0 & 0.0 & 0.0 & 0.0 & 0.0 & 0.0 & 0.0 & 0.0 & 0.0 & 0.0 \\
\hline & 0.20 & 60000 & 60.0 & 279 & 7.00 & 2.50 & 0.0 & 0.0 & 0.0 & 0.0 & 0.0 & 0.0 & 0.0 & 0.0 & 0.0 & 0.0 & 0.0 & 1 \\
\hline & 0.40 & 40000 & 40.0 & 245 & 6.00 & 2.45 & 1 & 0.0 & 0.0 & 0.0 & 0.0 & 0.0 & 0.0 & 0.0 & 1 & 0.0 & 0.0 & 0.0 \\
\hline & 0.60 & 18000 & 18.0 & 295 & 8.00 & 2.71 & 0.0 & 0.0 & 1 & 0.0 & 1 & 1 & 1 & 1 & 1 & 0.0 & 0.0 & 2.0 \\
\hline & 0.80 & 7000 & 7.0 & 300 & 9.00 & 3.00 & 1 & 1 & 0.0 & 1 & 0.0 & 2 & 0.0 & 1 & 1 & 1 & 1 & 0.0 \\
\hline & 1.00 & 200 & 0.2 & 100 & 3.00 & 3.00 & 0.0 & 0.0 & 1 & 0.0 & 1 & 0.0 & 0.0 & 0.0 & 1 & 0.0 & 0.0 & 0.0 \\
\hline & Total & & & 1489 & 34 & 2.34 & 3 & 3 & 2 & 2 & 3 & 3 & 2 & 3 & 4 & 3 & 3 & 3 \\
\hline
\end{tabular}


Table 2. Effect of six of Azotobacter chroococum strains combined with mineral Nfertilizer on growth and development of zucchini type summer squash (Cucurbita pepo L.) cv "Eskandarany" plants grown in 2008 and 2009 ..

\begin{tabular}{|c|c|c|c|c|c|c|c|c|c|}
\hline \multicolumn{2}{|c|}{ Treatments ${ }^{(2)}$} & \multicolumn{2}{|c|}{ Number of leaves } & \multicolumn{2}{|c|}{ Stem length } & \multicolumn{2}{|c|}{$\begin{array}{c}\text { Number of Female } \\
\text { flower }\end{array}$} & \multicolumn{2}{|c|}{$\begin{array}{c}\text { Number of male } \\
\text { flower }\end{array}$} \\
\hline \multirow{6}{*}{ 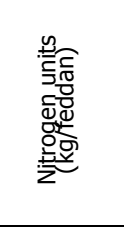 } & & 2008 & 2009 & 2008 & 2009 & 2008 & 2009 & 2008 & 2009 \\
\hline & 0 & 21.37 & 20.47 & 30.07 & 29.83 & 6.93 & 6.74 & 12.76 & 12.36 \\
\hline & 30 & 23.79 & 23.04 & 37.61 & 36.64 & 8.75 & 8.44 & 14.14 & 13.41 \\
\hline & 60 & 26.25 & 26.58 & 43.90 & 43.06 & 11.18 & 10.95 & 15.11 & 14.49 \\
\hline & 90 & 28.90 & 29.36 & 52.66 & 52.14 & 13.64 & 13.21 & 15.14 & 14.30 \\
\hline & 120 & 31.30 & 30.97 & 61.54 & 60.60 & 15.47 & 15.36 & 14.97 & 14.59 \\
\hline L.S.D. 0.05 & & 0.02 & 0.07 & 0.03 & 0.04 & 0.26 & 0.01 & 0.09 & 0.11 \\
\hline \multirow{7}{*}{ 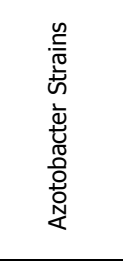 } & Control $^{-}$ & 25.16 & 25.00 & 40.36 & 39.58 & 9.84 & 9.53 & 15.50 & 15.01 \\
\hline & Control $^{+}$ & 25.92 & 25.51 & 44.46 & 43.58 & 10.55 & 10.40 & 14.56 & 14.04 \\
\hline & 3 & 26.16 & 25.85 & 44.96 & 43.98 & 10.82 & 10.70 & 14.40 & 13.76 \\
\hline & 4 & 26.40 & 26.21 & 45.34 & 44.74 & 11.25 & 11.02 & 14.30 & 13.65 \\
\hline & 5 & 26.58 & 26.45 & 46.54 & 45.88 & 11.55 & 11.28 & 14.20 & 13.52 \\
\hline & 6 & 26.87 & 26.73 & 47.02 & 46.26 & 11.95 & 11.60 & 14.16 & 13.47 \\
\hline & 7 & 27.16 & 26.85 & 47.42 & 47.16 & 12.40 & 12.05 & 13.86 & 13.36 \\
\hline L.S.D. 0.05 & & 0.023 & 0.076 & 0.071 & 0.052 & 0.260 & 0.009 & 0.089 & 0.104 \\
\hline \multirow{7}{*}{ 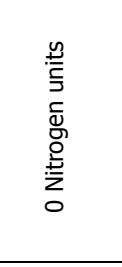 } & Control $^{-}$ & 19.50 & 19.00 & 25.00 & 24.70 & 5.00 & 4.75 & 14.00 & 13.27 \\
\hline & Control $^{+}$ & 21.00 & 20.25 & 31.00 & 30.30 & 6.50 & 6.25 & 13.00 & 12.60 \\
\hline & 3 & 21.30 & 20.25 & 30.00 & 29.60 & 6.50 & 6.35 & 13.00 & 12.50 \\
\hline & 4 & 21.75 & 20.75 & 30.00 & 30.20 & 7.25 & 7.25 & 12.50 & 12.00 \\
\hline & 5 & 21.80 & 20.75 & 31.00 & 30.70 & 7.50 & 7.35 & 12.50 & 12.30 \\
\hline & 6 & 22.00 & 21.03 & 31.50 & 31.00 & 7.75 & 7.50 & 12.30 & 12.03 \\
\hline & 7 & 22.25 & 21.25 & 32.00 & 32.30 & 8.00 & 7.75 & 12.00 & 11.80 \\
\hline \multirow{7}{*}{ 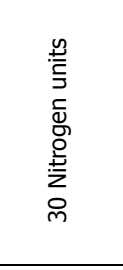 } & Control $^{-}$ & 22.80 & 22.00 & 33.00 & 31.70 & 7.00 & 6.50 & 15.00 & 14.50 \\
\hline & Control $^{+}$ & 23.50 & 22.25 & 36.00 & 34.60 & 8.00 & 7.75 & 14.50 & 13.80 \\
\hline & 3 & 23.70 & 22.70 & 36.60 & 35.30 & 8.00 & 7.90 & 14.00 & 13.50 \\
\hline & 4 & 23.75 & 23.00 & 36.90 & 36.00 & 8.75 & 8.60 & 14.00 & 13.30 \\
\hline & 5 & 24.00 & 23.50 & 40.00 & 39.30 & 9.00 & 8.80 & 14.00 & 13.00 \\
\hline & 6 & 24.25 & 23.80 & 40.30 & 39.70 & 10.00 & 9.50 & 14.00 & 13.00 \\
\hline & 7 & 24.50 & 24.00 & 40.50 & 39.90 & 10.50 & 10.00 & 13.50 & 12.80 \\
\hline \multirow{7}{*}{ 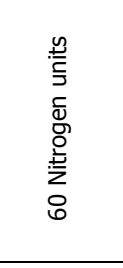 } & Control $^{-}$ & 25.25 & 25.00 & 39.00 & 38.50 & 9.50 & 9.40 & 16.50 & 16.40 \\
\hline & Control $^{+}$ & 25.80 & 25.53 & 43.50 & 42.70 & 11.00 & 10.50 & 15.00 & 14.50 \\
\hline & 3 & 26.00 & 26.50 & 44.30 & 43.20 & 11.00 & 11.00 & 15.00 & 14.30 \\
\hline & 4 & 26.25 & 26.80 & 44.70 & 43.70 & 11.25 & 11.00 & 15.00 & 14.20 \\
\hline & 5 & 26.33 & 27.00 & 44.90 & 43.90 & 11.50 & 11.25 & 15.00 & 14.00 \\
\hline & 6 & 26.80 & 27.75 & 45.30 & 44.60 & 11.50 & 11.50 & 15.00 & 14.00 \\
\hline & 7 & 27.30 & 27.50 & 45.60 & 44.80 & 12.50 & 12.00 & 14.30 & 14.00 \\
\hline \multirow{7}{*}{ 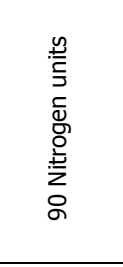 } & Control $^{-}$ & 27.50 & 28.50 & 46.80 & 45.70 & 12.50 & 12.00 & 16.00 & 15.50 \\
\hline & Control $^{+}$ & 28.50 & 29.00 & 51.50 & 50.30 & 13.00 & 12.50 & 15.00 & 14.30 \\
\hline & 3 & 28.80 & 29.00 & 52.50 & 51.30 & 13.25 & 13.00 & 15.00 & 14.00 \\
\hline & 4 & 29.00 & 29.50 & 53.30 & 52.60 & 13.50 & 13.00 & 15.50 & 14.30 \\
\hline & 5 & 29.25 & 29.75 & 54.50 & 54.00 & 14.00 & 13.50 & 14.50 & 14.00 \\
\hline & 6 & 29.50 & 29.80 & 54.80 & 54.20 & 14.50 & 14.00 & 15.00 & 14.00 \\
\hline & 7 & 29.75 & 30.00 & 55.20 & 56.90 & 14.75 & 14.50 & 15.00 & 14.00 \\
\hline \multirow{7}{*}{ 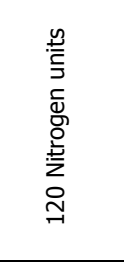 } & Control $^{-}$ & 30.75 & 30.50 & 58.00 & 57.30 & 15.20 & 15.00 & 16.00 & 15.40 \\
\hline & Control $^{+}$ & 30.80 & 30.50 & 60.30 & 60.00 & 14.25 & 15.00 & 15.30 & 15.00 \\
\hline & 3 & 31.00 & 30.80 & 61.40 & 60.50 & 15.35 & 15.25 & 15.00 & 14.50 \\
\hline & 4 & 31.24 & 31.00 & 61.80 & 61.20 & 15.50 & 15.25 & 14.50 & 14.43 \\
\hline & 5 & 31.50 & 31.25 & 62.30 & 61.50 & 15.75 & 15.50 & 15.00 & 14.30 \\
\hline & 6 & 31.80 & 31.25 & 63.20 & 61.80 & 16.00 & 15.50 & 14.50 & 14.30 \\
\hline & 7 & 32.00 & 31.50 & 63.80 & 61.90 & 16.25 & 16.00 & 14.50 & 14.20 \\
\hline L.S.D. 0.0 & & 0.05 & 0.17 & 0.16 & 0.12 & 0.58 & 0.02 & 0.20 & 0.23 \\
\hline
\end{tabular}

${ }^{(1)}$ Control $^{-}$and Control $^{+}=$without inoculation or inoculated with Azotobacter wild strains, 3 to 7 are induce Azotobacter chroococum mutants

(2) Interaction of nitrogen application rate and Azotobacter strains was significant. 
Table 3. Effect of six of Azotobacter strains combined with mineral $\mathrm{N}$-fertilizer on number of fruits, eqarly and total yield zucchini type summer squash (Cucurbita pepo L.) Cv "Eskandarany" plants grown in 2008 and 2009 .

\begin{tabular}{|c|c|c|c|c|c|c|c|}
\hline \multirow{2}{*}{\multicolumn{2}{|c|}{ Treatments }} & \multicolumn{2}{|c|}{ Number of fruits } & \multicolumn{2}{|c|}{ Early yield } & \multicolumn{2}{|c|}{ Total yield (ton/Feddan) } \\
\hline & & 2008 & 2009 & 2008 & 2009 & 2008 & 2009 \\
\hline \multirow{5}{*}{ 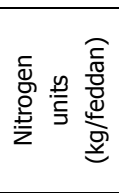 } & 0 & 3.300 & 3.164 & 1.121 & 1.108 & 3.383 & 3.234 \\
\hline & 30 & 4.021 & 3.893 & 1.504 & 1.476 & 4.627 & 4.481 \\
\hline & 60 & 5.129 & 5.000 & 2.071 & 2.034 & 6.055 & 5.899 \\
\hline & 90 & 6.071 & 5.950 & 2.671 & 2.591 & 7.771 & 7.587 \\
\hline & 120 & 6.436 & 6.343 & 2.819 & 2.720 & 8.587 & 8.424 \\
\hline L.S.D. 0.05 & & 0.02 & 0.01 & 0.001 & 0.021 & 0.031 & 0.004 \\
\hline \multirow{7}{*}{ 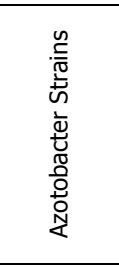 } & Control $^{-}$ & 4.180 & 4.070 & 1.450 & 1.415 & 4.856 & 4.742 \\
\hline & Control $^{+}$ & 4.760 & 4.640 & 1.870 & 1.834 & 5.713 & 5.555 \\
\hline & 3 & 4.920 & 4.790 & 1.950 & 1.884 & 5.921 & 5.768 \\
\hline & 4 & 5.040 & 4.930 & 2.046 & 2.005 & 6.151 & 6.001 \\
\hline & 5 & 5.170 & 5.040 & 2.160 & 2.100 & 6.352 & 6.175 \\
\hline & 6 & 5.330 & 5.200 & 2.330 & 2.269 & 6.630 & 6.444 \\
\hline & 7 & 5.540 & 5.420 & 2.456 & 2.393 & 6.969 & 6.789 \\
\hline L.S.D. 0.05 & & 0.018 & 0.007 & 0.001 & 0.021 & 0.030 & 0.004 \\
\hline \multirow{7}{*}{ 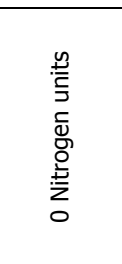 } & Control $^{-}$ & 2.100 & 2.000 & 0.300 & 0.295 & 1.739 & 1.650 \\
\hline & Control $^{+}$ & 3.000 & 2.850 & 1.000 & 0.995 & 2.999 & 2.836 \\
\hline & 3 & 3.250 & 3.200 & 1.150 & 1.090 & 3.311 & 3.245 \\
\hline & 4 & 3.500 & 3.300 & 1.200 & 1.192 & 3.596 & 3.383 \\
\hline & 5 & 3.500 & 3.350 & 1.300 & 1.330 & 3.649 & 3.476 \\
\hline & 6 & 3.750 & 3.600 & 1.400 & 1.389 & 4.013 & 3.834 \\
\hline & 7 & 4.000 & 3.850 & 1.500 & 1.465 & 4.375 & 4.211 \\
\hline \multirow{7}{*}{ 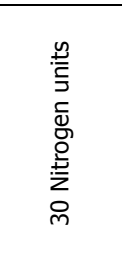 } & Control $^{-}$ & 3.200 & 3.000 & 1.150 & 1.100 & 3.296 & 3.186 \\
\hline & Control $^{+}$ & 3.500 & 3.400 & 1.400 & 1.382 & 3.938 & 3.810 \\
\hline & 3 & 4.000 & 3.850 & 1.350 & 1.341 & 4.605 & 4.430 \\
\hline & 4 & 4.000 & 3.900 & 1.550 & 1.535 & 4.625 & 4.483 \\
\hline & 5 & 4.250 & 4.100 & 1.450 & 1.440 & 4.968 & 4.780 \\
\hline & 6 & 4.400 & 4.250 & 1.700 & 1.685 & 5.198 & 5.000 \\
\hline & 7 & 4.800 & 4.750 & 1.930 & 1.850 & 5.760 & 5.676 \\
\hline \multirow{7}{*}{ 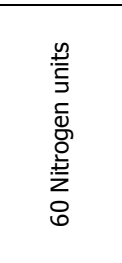 } & Control $^{-}$ & 4.200 & 4.100 & 1.500 & 1.480 & 4.735 & 4.610 \\
\hline & Control $^{+}$ & 5.100 & 5.000 & 1.850 & 1.810 & 5.941 & 5.813 \\
\hline & 3 & 5.000 & 4.800 & 1.950 & 1.920 & 5.733 & 5.588 \\
\hline & 4 & 5.100 & 5.000 & 1.900 & 1.880 & 5.993 & 5.863 \\
\hline & 5 & 5.300 & 5.150 & 2.250 & 2.210 & 6.261 & 6.070 \\
\hline & 6 & 5.500 & 5.350 & 2.450 & 2.385 & 6.669 & 6.460 \\
\hline & 7 & 5.700 & 5.600 & 2.600 & 2.550 & 7.054 & 6.888 \\
\hline \multirow{7}{*}{ 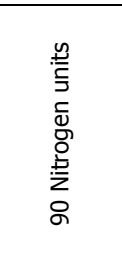 } & Control $^{-}$ & 5.200 & 5.150 & 1.750 & 1.700 & 6.370 & 6.290 \\
\hline & Control $^{+}$ & 5.900 & 5.750 & 2.450 & 2.395 & 7.339 & 7.130 \\
\hline & 3 & 6.000 & 5.800 & 2.550 & 2.420 & 7.500 & 7.229 \\
\hline & 4 & 6.200 & 6.100 & 2.800 & 2.730 & 7.983 & 7.824 \\
\hline & 5 & 6.300 & 6.200 & 2.950 & 2.810 & 8.190 & 8.029 \\
\hline & 6 & 6.400 & 6.300 & 3.050 & 2.985 & 8.400 & 8.230 \\
\hline & 7 & 6.500 & 6.350 & 3.150 & 3.100 & 8.613 & 8.375 \\
\hline \multirow{7}{*}{ 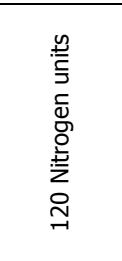 } & Control $^{-}$ & 6.200 & 6.100 & 2.550 & 2.500 & 8.138 & 7.976 \\
\hline & Control $^{+}$ & 6.300 & 6.200 & 2.650 & 2.590 & 8.348 & 8.184 \\
\hline & 3 & 6.350 & 6.300 & 2.750 & 2.650 & 8.454 & 8.348 \\
\hline & 4 & 6.400 & 6.350 & 2.780 & 2.690 & 8.560 & 8.454 \\
\hline & 5 & 6.500 & 6.400 & 2.850 & 2.710 & 8.694 & 8.520 \\
\hline & 6 & 6.600 & 6.500 & 3.050 & 2.900 & 8.869 & 8.694 \\
\hline & 7 & 6.700 & 6.550 & 3.100 & 3.000 & 9.045 & 8.794 \\
\hline L.S.D. 0.05 & & 0.04 & 0.02 & 0.002 & 0.046 & 0.068 & 0.010 \\
\hline
\end{tabular}

(1) Control $^{-}$and Control $^{+}=$without inoculation or inoculated with Azotobacter wild strains, 3 to 7 are induce Azotobacter chroococum mutants

(2) Interaction of nitrogen application rate and Azotobacter strains was significant. 
Table 4. Effect of six of Azotobacter strains combined with mineral $\mathrm{N}$-fertilizer on fruit Length, diameter and weight of zucchini type summer squash (Cucurbita pepo L.) CV "Eskandarany" plants grown in 2008 and 2009.

\begin{tabular}{|c|c|c|c|c|c|c|c|}
\hline \multicolumn{2}{|c|}{ Treatments } & \multicolumn{2}{|c|}{ Fruit length $(\mathrm{cm})$} & \multicolumn{2}{|c|}{ Fruit diameter $(\mathrm{cm})$} & \multicolumn{2}{|c|}{ Fruit weight (g) } \\
\hline & & 2008 & 2009 & 2008 & 2009 & 2008 & 2009 \\
\hline \multirow{5}{*}{ 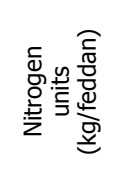 } & 0 & 7.759 & 7.657 & 2.025 & 1.943 & 80.93 & 80.65 \\
\hline & 30 & 8.937 & 8.861 & 2.514 & 2.469 & 91.57 & 91.30 \\
\hline & 60 & 9.714 & 9.650 & 2.829 & 2.804 & 94.50 & 94.17 \\
\hline & 90 & 11.802 & 11.739 & 3.385 & 3.359 & 102.21 & 101.83 \\
\hline & 120 & 13.195 & 13.119 & 3.929 & 3.904 & 106.71 & 106.23 \\
\hline L.S.D. 0.05 & & 0.070 & 0.062 & 0.013 & 0.006 & 0.07 & 0.09 \\
\hline \multirow{7}{*}{ 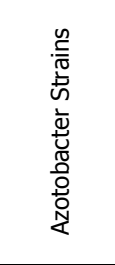 } & Control $^{-}$ & 9.153 & 9.130 & 2.536 & 2.512 & 88.38 & 88.11 \\
\hline & Control $^{+}$ & 9.860 & 9.766 & 2.780 & 2.742 & 93.74 & 93.42 \\
\hline & 3 & 9.996 & 9.920 & 2.820 & 2.778 & 94.74 & 94.40 \\
\hline & 4 & 10.356 & 10.320 & 2.981 & 2.946 & 95.74 & 95.47 \\
\hline & 5 & 10.490 & 10.430 & 3.057 & 3.006 & 96.47 & 96.14 \\
\hline & 6 & 10.962 & 10.843 & 3.136 & 3.092 & 97.92 & 97.48 \\
\hline & 7 & 11.153 & 11.026 & 3.244 & 3.194 & 99.30 & 98.84 \\
\hline L.S.D. 0.05 & & 0.048 & 0.028 & 0.008 & 0.006 & 0.066 & 0.082 \\
\hline \multirow{7}{*}{ 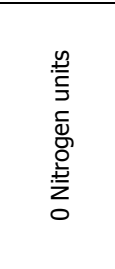 } & Control $^{-}$ & 6.700 & 6.800 & 1.800 & 1.750 & 66.30 & 66.00 \\
\hline & Control $^{+}$ & 7.700 & 7.500 & 1.950 & 1.850 & 80.00 & 79.60 \\
\hline & 3 & 7.730 & 7.700 & 1.980 & 1.900 & 81.50 & 81.20 \\
\hline & 4 & 7.780 & 7.750 & 2.000 & 1.980 & 82.20 & 82.27 \\
\hline & 5 & 7.900 & 7.850 & 2.097 & 2.000 & 83.40 & 83.00 \\
\hline & 6 & 8.200 & 8.000 & 2.150 & 2.050 & 85.60 & 85.20 \\
\hline & 7 & 8.300 & 8.000 & 2.200 & 2.070 & 87.50 & 87.30 \\
\hline \multirow{7}{*}{ 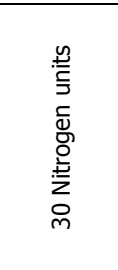 } & Control $^{-}$ & 7.800 & 7.750 & 2.000 & 2.000 & 82.40 & 82.23 \\
\hline & Control $^{+}$ & 8.800 & 8.780 & 2.400 & 2.350 & 90.00 & 89.70 \\
\hline & 3 & 8.900 & 8.800 & 2.450 & 2.370 & 92.10 & 92.00 \\
\hline & 4 & 8.950 & 8.900 & 2.500 & 2.470 & 92.50 & 92.20 \\
\hline & 5 & 9.100 & 9.000 & 2.700 & 2.600 & 93.50 & 93.30 \\
\hline & 6 & 9.310 & 9.167 & 2.750 & 2.710 & 94.50 & 94.10 \\
\hline & 7 & 9.700 & 9.630 & 2.800 & 2.780 & 96.00 & 95.60 \\
\hline \multirow{7}{*}{ 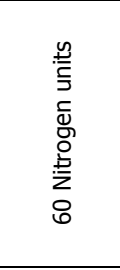 } & Control $^{-}$ & 8.900 & 8.850 & 2.430 & 2.410 & 90.20 & 90.00 \\
\hline & Control $^{+}$ & 9.100 & 9.000 & 2.700 & 2.700 & 93.20 & 93.00 \\
\hline & 3 & 9.150 & 9.100 & 2.710 & 2.700 & 93.60 & 93.10 \\
\hline & 4 & 9.500 & 9.450 & 2.800 & 2.750 & 94.00 & 93.80 \\
\hline & 5 & 9.550 & 9.500 & 2.850 & 2.820 & 94.50 & 94.30 \\
\hline & 6 & 10.800 & 10.750 & 3.010 & 3.000 & 97.00 & 96.60 \\
\hline & 7 & 11.000 & 10.900 & 3.300 & 3.250 & 99.00 & 98.40 \\
\hline \multirow{7}{*}{ 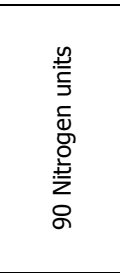 } & Control $^{-}$ & 10.300 & 10.250 & 2.900 & 2.900 & 98.00 & 97.70 \\
\hline & Control $^{+}$ & 10.700 & 10.600 & 2.950 & 2.930 & 99.50 & 99.20 \\
\hline & 3 & 11.000 & 10.900 & 3.020 & 3.000 & 100.00 & 99.70 \\
\hline & 4 & 12.250 & 12.250 & 3.603 & 3.550 & 103.00 & 102.60 \\
\hline & 5 & 12.400 & 12.370 & 3.620 & 3.610 & 104.00 & 103.60 \\
\hline & 6 & 12.900 & 12.800 & 3.750 & 3.700 & 105.00 & 104.50 \\
\hline & 7 & 13.067 & 13.000 & 3.850 & 3.820 & 106.00 & 105.50 \\
\hline \multirow{7}{*}{ 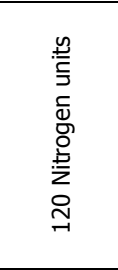 } & Control $^{-}$ & 12.067 & 12.000 & 3.550 & 3.500 & 105.00 & 104.60 \\
\hline & Control $^{+}$ & 13.000 & 12.950 & 3.900 & 3.880 & 106.00 & 105.60 \\
\hline & 3 & 13.200 & 13.100 & 3.940 & 3.920 & 106.50 & 106.00 \\
\hline & 4 & 13.300 & 13.250 & 4.000 & 3.980 & 107.00 & 106.50 \\
\hline & 5 & 13.500 & 13.430 & 4.020 & 4.000 & 106.97 & 106.50 \\
\hline & 6 & 13.600 & 13.500 & 4.020 & 4.000 & 107.50 & 107.00 \\
\hline & 7 & 13.700 & 13.600 & 4.070 & 4.050 & 108.00 & 107.40 \\
\hline L.S.D. 0.05 & & 0.108 & 0.062 & 0.019 & 0.013 & 0.15 & 0.18 \\
\hline
\end{tabular}

(1) Control $^{-}$and Control $^{+}=$without inoculation or inoculated with Azotobacter wild strains, 3 to 7 are induce Azotobacter chroococum mutants

${ }^{(2)}$ Interaction of nitrogen application rate and Azotobacter strains was significant. 


\section{REFERENCES}

1. Abd El-Fattah, M.A. and M.E. Sorial. 2000. Sex expression and productivity responses of summer squash to biofertilizer application under different nitrogen levels. Zagzig J. Agric. Res.27 (2): 255-281.

2. Abdalla, A.M., F.A Rizk and S.M. Adam. 2001. The productivity of pepper plants as influenced by some bio-fertilizer treatments under plastic house conditions. Bull.Fac.Agric.Cairo Univ.52 (4): 625:639.

3. Abdel-Raheem ,T.A., R .H. Ragab, O. F. Dakhly and E.A. Eid. 1995. Improvement of Azotobacter vindndii efficiently for nitrogen fixation through mutateim induction and conjugation .Egypt J. Appl. Sci.,10:753-771.

4. Adam, S.M, A.M. Abdalla and F.A. Risk. 2002. Effect of the interaction between the mineral and bio-fertilizer on the productivity of cantaloupe (Cucumis melo L.) under the newly reclaimed soils conditions. Egypt. J. Hort. 29(2): 301-315.

5. Bishop, P.E., D.M.S. Jarlenski and D.R. Hetherington. 1980. Evidence for an alternative nitrogen fixation system in Azotobacter vinelndii Proc.Nath. Acad. Sci., U.S,A. 77:7342-7346.

6. Bloembberg, G.V and Bjj. Lugtenberg. 2001. Molecular basis of plant growth promotion and biocontrol by rhizobacteria. Current opinion in plant Biology 4: 343350.

7. Bochow, H. and S. Dolej. 1999. Mechanisms of tolerance induction in plants by roots colonizing Basillus subtilis isolates.In : Modern fungicides and antifungal compound II. Intercept LTD. Po Box 716. Andover, Hampshire SP101 Yg. UK, 1999: 411-416.

8. Dakhly O.F. and Y.T. Abedel-Mageed. 1997. Estimation of Effectiveness of Azotobacter chroococum trransformants on growth and yield of some vegetable crops .Egypt J.Genet, Cytol. 26:73-88.

9. Dakhly O.F., M.A.H. Mahmoud and F.A.S. Nassif. 1998. Studies on the efficiency of Azotobacter vinelndii and Bacilliies megathericm var. phosphaticium wild types and their UV induced mutants on sorghum .Assiut J. Agric. Sci. (29):75-89.

10. De Silva A, K. Petterson, C. Rothrock and J. Moore. 2000. Growth promotion of highbush blueberry by fungal and bacterial inoculants. HortScience 35:1228-1230.

11. El- Karamany M.F., M.K.A. Ahmed, A.A. Bahr and M.O. kabesh.. 2000. Utilization of bio-fertilizers in field crop production. Egypt.J .Appl. Sci.15 (11):137-155.

12. El-Metwaly, I.M. 1998. Effect of herbicides and bio-fertilization on growth and yield of wheat under different nitrogen fertilization levels. Ph.D.Thesis, Mansoura Univ. 
13. Goicoechea, N., K. Dolezal, M.C. Antolin, M. Strand and M. Sanchez. 1995. Influence of Mycorrhizae and Rhizobium on cytokinin content in drought-stressed alfalfa. J. Exp. Botany, 46 (291):1543-1549.

14. Gomez K. A. and A. A. Gomez. 1984. Statistical procedure for Agricultural Research 2nd (ed.) John Wily \& Sons New York.

15. Hassan, E.A., O.F. Dakhly and F.A.S. Nassif. 2000. Increasing Yield of squash plants through inoculation by mutants and hybrid of two Azotobacter species. Procceed 2 nd Arab Cong. Genet. Biotec.2000, 131-150.

16. Holliday .R. 1960. A new method for the identification of Biochemical mutants of microorganisms In."Mycogentics" John Wiles and Sons London .P.41.

17. Kumar, $V$ and $\mathrm{N}$.Norula. 1999. Solubilization of inorganic phosphates and growth emergence of wheat as affected by Azotobacter chroococum mutants. Biol. Fertil. Soils (28): 301-305.

18. Lau, T. and A.G. Stephenson. 1993. Effects of soil nitrogen on pollen production, pollen grain size and pollen performance in Cucurbita pepo. Amer.J. Bot. 80 (7): 763-768.

19. Mahmoud, M.A. 2000. Growth emergence of maize in response to inoculation with wild type and induced biochemical mutants-solubilizing Azotobacter chroococum .J. Agric. Mansoura Univ., 25(5): 25555-2567.

20. Mohamed, M.F. 1996. Phenotypic variability and selection for predominant pistillate flower expression in zucchini-type summer squash (Cucurbita pepo L.) "Eskandarani". First Egyptian-Hungarian Horticultural Conference, sept. 1996. Vol. 2, p.14-162, Kafr El-sheikh, Tanta univ., Egypt.

21. Mchenney, D. and T. Melton. 1986. Isolation and characterization of ack and pta mutations in Azotobacter vinelndii affecting Acetotee-Glucose Diauxis. J. Bact.164:6-12.

22. Neyra, C .A. and J .Dobereiner. 1977. Nitrogen fixation associated with grasses .Adv. Agron., 29: 1-38.

23. Noel, T.C., C. Sheng, C.K. Yost, R.P. Pharis and M.E. Hynes. 1996. Rhizobium leguminosarum as a plant growth promoting Rhizobacterium, direct growth promotion of canola and lettuce. Can. J. Microbiol. 24 (3): 279-283.

24. Rai, M.K., 2006. Handbook of microbial biofertilizers. Food Products Press, an imprint of The Haworth Press, Inc, Binghamton, New York.

25. Refai, E.F.S., M.F. Mohamed. 2009. Population and single plant-derived inbred line analysis for sex expression in summer squash (Cucurbita pepo L.) cv. Eskandrani. Assuit University Bull. Environ Res. Vol. 12 No. 1, March 2009. 
254 GROWTH AND YIELD OF ZUCCHINI TYPE SUMMER SQUASH (CUCURBITA PEPO L.) FERTILIZED BY COMBINED AZOTOBACTER CHROOCOCUM MUTANTS AND MINERAL N-FERTILIZATION

26. Shehata, M.M. and S.A. El- Khawas. 2003. Effect of two biofertilizers on growth parameters, yield characters, nitrogenous components, nucleic acids content, minerals, oil content, protein profiles and DNA banding pattern of sunflower (Helianthus annus L. cv. vedock) yield. Pakistan J. Biol. Sci., 6(14): 1257-1268.

27. Strandberg ,G.W. and P.W. Wilson. 1968. Formation of the nitrogen fixing enzyme system in Azotobacter vinelndii .Con. J.Microbial.,14: 25-31.

28. Sudhakar, P, GN. Chattopadhyay, Sk. Gangwar and JK. Ghosh. 2000.Effect of foliar application of Azotobacter, Azospirillum and Beijerinckia on leaf yield and quality of of mulberry (Morus alba). The Journal of Agricultural Science (2000), 134: 227-234

29. Swiader, J.M., S.K. Sipp and R.E. Brown. 1994. Pumpkin growth, flowering and fruiting response to nitrogen and potassium sprinkler fertigation in sandy soil. J. Amer. Soc. Hort. Sci., 119:414-419.

30. Yin, T. and J.A. Quinn. 1995. Tests of an mechanistic model of one hormone regulating both sexes in Cucumis sativus. Amer.J.Bot.82 (12): 1537-1546. 


\section{نمو ومحصول الكوسة (كيوكربيتا بيبو) طراز زوكينى المخصبه باستخلام}

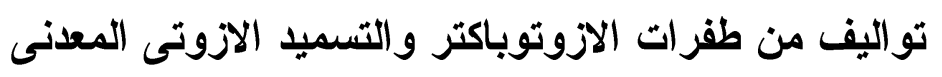

عماد الاين فؤاد سيد 13

$$
\text { r. . مركز البحوث النزراعيه - معهة بحوث البساتين - محطة اسيوط }
$$

أجريت هذه الدراسة خلال موسمين شتويين متتالين لعامي 2008 و 2009 بغرض دراسة

استجابة الكوسة صنف اسكندر انى لتوليفة من الازوت المعدني و التسميد الحيوي باستخدام طفر الترات

جديدة من الازوتوباكتر كروكوكم و التعرف على كفاءة هذه الطفرات من خلال تثيت الأبت النتروجين

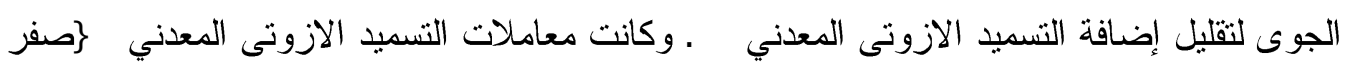

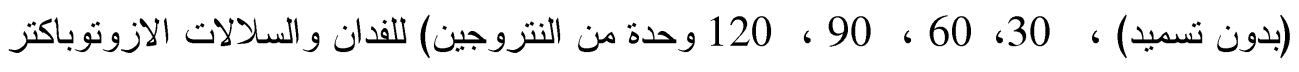

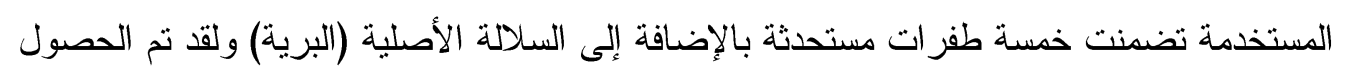

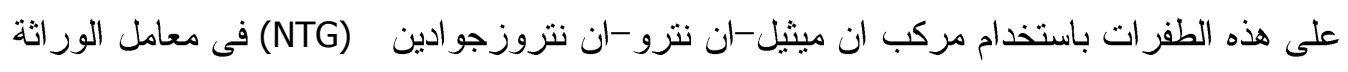

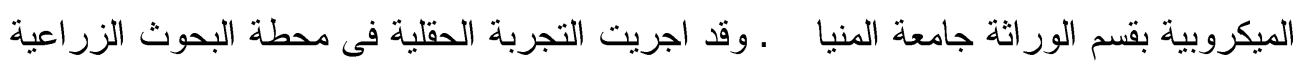

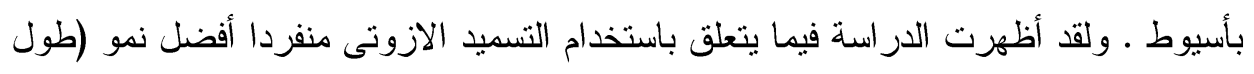

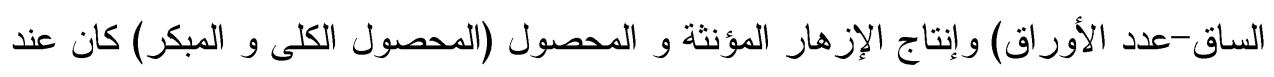

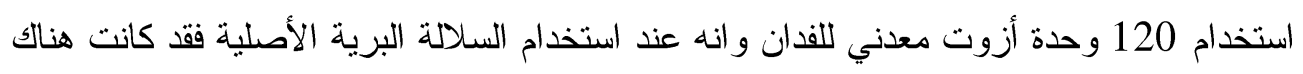

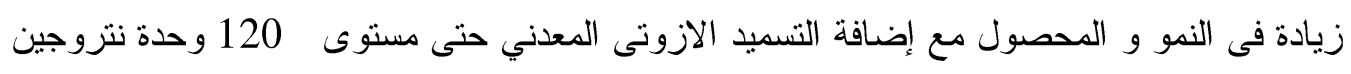

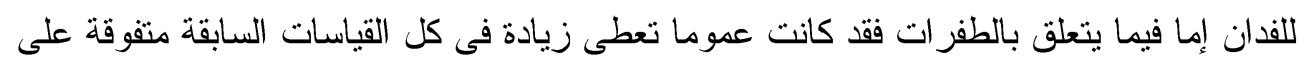

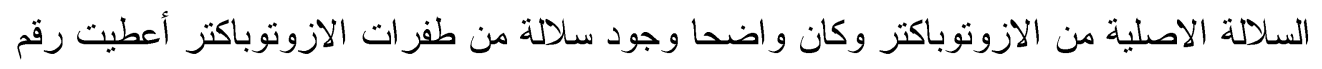

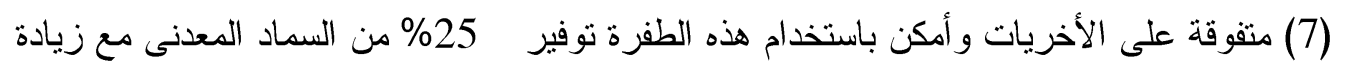

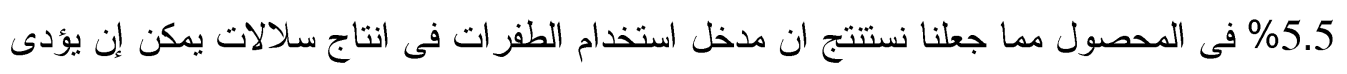

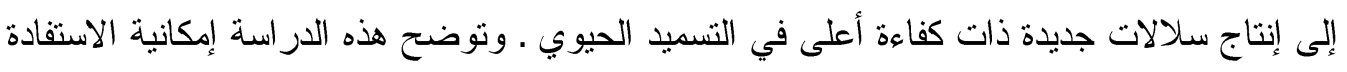

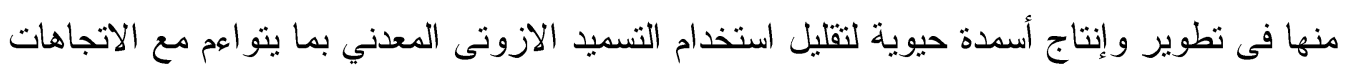

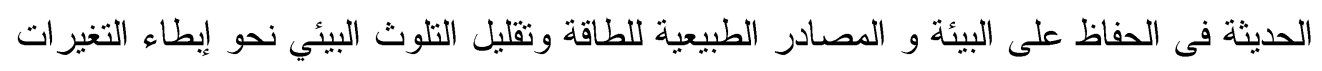
المناخية فى الاتجاه الغير مرغوب و إضر ارها على بيئة الكائنات الحية. 